\title{
Invariance principle for a Brownian motion with large drift in a white noise environment
}

\author{
Kiyoshi KaWAZU and Hiroshi TanaKa \\ (Received November 14, 1996)
}

\begin{abstract}
This paper discusses an invariance principle for a Brownian motion with drift coefficient $\kappa / 4$ in a white noise environment under the assumption that $\kappa$ is large. Our method clarifies the relation between the environment-wise invariance principle discussed in [7] and the present result (the invariance principle in random environment).
\end{abstract}

\section{Introduction}

Let $W$ be the space of continuous functions on $\mathbf{R}$ vanishing at 0 that is equipped with the Wiener measure $P$. For an element $w \in W$ let us denote by $w_{\kappa}$ the element of $W$ defined by $w_{\kappa}(x)=w(x)-(\kappa x / 2)$ where $\kappa$ is a given positive constant. For $w \in W, P_{w}$ denotes the probability measure on $\Omega=$ $C[0, \infty)$ such that $\mathbf{X}_{x}=\left\{\omega(t), t \geq 0, P_{w}\right\}$ is a diffusion process with generator

$$
\mathscr{L}_{w}=\frac{1}{2} e^{w_{\kappa}}(x) \frac{d}{d x}\left(e^{-w_{\kappa}}(x) \frac{d}{d x}\right)
$$

starting at 0 , where $\omega(t)$ is the value of a function $\omega(\in \Omega)$ at time $t$. We regard $\omega(t)$ as a process defined on the probability space $\{W \times \Omega, \mathscr{P}\}$ where $\mathscr{P}(d w d \omega)=P(d w) P_{w}(d \omega)$. Then symbolically

$$
d \omega(t)=d B(t)+\frac{\kappa}{4} d t-\frac{1}{2} w^{\prime}(\omega(t)) d t
$$

where $B(t)$ is a standard Brownian motion independent of the white noise $\left\{w^{\prime}(x)\right\}$. We call the process $\mathbf{X}=\{\omega(t), t \geq 0, \mathscr{P}\}$ a Brownian motion with drift in a white noise environment; in [2] [6] [7] it is called a diffusion process in a Brownian environment with drift. The present authors obtained some limit theorems for $\mathbf{X}$ in [2] (see [8] for further results; see also [6] for a brief survey on related problems), which are analogous to those of [3] and [5]; however, some problems remain open. The present paper is a continuation of [7] and

1991 Mathematics Subject Classification. 60J60

Key words and phrases. Invariance principle, Brownian motion, Random environment. 
discusses the central limit theorem, or more precisely speaking, invariance principle in random environment in the case $\kappa>2$.

We set

$$
\begin{aligned}
& M_{x}(=M(x))=2 \int_{0}^{x} d y \int_{-\infty}^{y} e^{w_{\kappa}(y)-w_{\kappa}(z)} d z, x \in \mathbf{R}, \\
& \mu(t)=\text { the inverse function of }\left\{M_{x}, x \in \mathbf{R}\right\}, t \in \mathbf{R}, \\
& T_{x}(=T(x))=\inf \{t>0: \omega(t)>x\}, x \geq 0 .
\end{aligned}
$$

We use also the following notation:

$$
\begin{aligned}
& \bar{\omega}(t)=\max \{\omega(s): 0 \leq s \leq t\}, \underline{\omega}(t)=\inf \{\omega(s): s \geq t\},(\omega \in \Omega), \\
& \gamma=(\kappa-1) / 2, m=4 /(\kappa-1)=2 / \gamma \\
& A=64(\kappa-1)^{-2}(\kappa-2)^{-1}=16 \gamma^{-2}(2 \gamma-1)^{-1} \\
& B=64(\kappa-1)^{-3}(\kappa-2)^{-1}=8 \gamma^{-3}(2 \gamma-1)^{-1} \\
& C=A+B=64 \kappa(\kappa-1)^{-3}(\kappa-2)^{-1}=8(2 \gamma+1) \gamma^{-3}(2 \gamma-1)^{-1}
\end{aligned}
$$

The following theorem was proved in [7].

THEOREM A (Environment-wise invariance principle, see [7]). When $\kappa>2$, we have the following:

(i) For almost all $w \in W$ with respect to $P$, the process

$$
\left\{\frac{T_{\lambda x}-M_{\lambda x}}{\sqrt{A \lambda}}, x \geq 0, P_{w}\right\}
$$

converges in law to a Brownian motion as $\lambda \rightarrow \infty$ (in the sense of convergence of probability measures on the Skorohod space).

(ii) For almost all $w$, the process

$$
\left\{\frac{\omega(\lambda t)-\mu(\lambda t)}{\sqrt{m^{-3} A \lambda}}, t \geq 0, P_{w}\right\}
$$

converges in law to a Brownian motion as $\lambda \rightarrow \infty$ (in the sense of convergence of probability measures on $C[0, \infty)$ ). The same is true when $\omega(\lambda t)$ is replaced by either of $\bar{\omega}(\lambda t)$ and $\underline{\omega}(\lambda t)$.

Our main theorems are the following ( $\kappa>2$ is assumed throughout).

THEOREM 1. (i) The process

$$
\left\{\frac{M_{\lambda x}-\lambda m x}{\sqrt{B \lambda}}, x \in \mathbf{R}, P\right\}
$$

converges in law to a Brownian motion as $\lambda \rightarrow \infty$. 
(ii) The process

$$
\left\{\frac{\mu(\lambda t)-\lambda m^{-1} t}{\sqrt{m^{-3} B \lambda}}, t \in \mathbf{R}, P\right\}
$$

converges in law to a Brownian motion as $\lambda \rightarrow \infty$.

THEOREM 2 (Invariance principle in random environment). (i) The process

$$
\left\{\frac{T_{\lambda x}-\lambda m x}{\sqrt{C \lambda}}, x \geq 0, \mathscr{P}\right\}
$$

converges in law to a Brownian motion as $\lambda \rightarrow \infty$.

(ii) The process

$$
\left\{\frac{\omega(\lambda t)-\lambda m^{-1} t}{m^{-3} C \lambda}, t \geq 0, \mathscr{P}\right\}
$$

converges in law to a Brownian motion as $\lambda \rightarrow \infty$. The same is true when $\omega(\lambda t)$ is replaced by either of $\bar{\omega}(\lambda t)$ and $\underline{\omega}(\lambda t)$.

As in [7] we introduce a one parameter family of measure preserving transformations $\theta_{t}, t \in \mathbf{R}$, on $(W, P)$ defined by $\left(\theta_{t} w\right)(x)=w(x+t)-w(t)$, $x \in \mathbf{R}$. Clearly $\theta_{t} \theta_{s}=\theta_{t+s}$ and $\left\{\theta_{t}\right\}$ is ergodic. Set

$$
f_{0}(w)=\int_{-\infty}^{0} e^{-w_{\kappa}(t)} d t
$$

Then $\theta_{t} f_{0} \equiv f_{0}\left(\theta_{t} w\right)=\int_{-\infty}^{t} e^{w_{\kappa}(t)-w_{x}(s)} d s$ and we have the following (see [7]):

$$
E_{w}\left\{T_{x}\right\}=M_{x}=2 \int_{0}^{x} \theta_{y} f_{0} d y
$$

the first equality of $(0.2)$ holds for $x \geq 0$ and the second one holds for $x \in \mathbf{R}$.

$$
\begin{gathered}
\operatorname{Var}_{w}\left\{T_{x}\right\}=8 \int_{0}^{x} \theta_{y} g d y \text { for } x \geq 0 \quad\left(g(w)=\int_{-\infty}^{0} e^{-w_{x}(t)}\left(\theta_{t} f_{0}\right)^{2} d t\right) . \\
E\left\{f_{0}\right\}=\gamma^{-1}, E\left\{f_{0}^{2}\right\}=2 \gamma^{-1}(2 \gamma-1)^{-1} \\
E\left\{\operatorname{Var}_{w}\left(T_{x}\right)\right\}=A x \text { for } x \geq 0 . \\
\operatorname{Var}\left\{M_{x}\right\}=B x+O(1), x \rightarrow \infty,(\operatorname{Var}=\text { variance }) .
\end{gathered}
$$

It was also observed in [7] that

$$
d \theta_{t} f_{0}=\theta_{t} f_{0} d w(t)-\left(\gamma \theta_{t} f_{0}-1\right) d t
$$


so $\theta_{t} f_{0}$ is a stationary diffusion process obtained as the unique stationary positive solution of the stochastic differential equation (0.7). Therefore

$$
\theta_{t} f_{0}-f_{0}=\int_{0}^{t} \theta_{s} f_{0} d w(s)-\lambda \int_{0}^{t} \theta_{s} f d s
$$

where $f=f_{0}-\gamma^{-1}$.

\section{Proof of Theorem 1}

For the proof of Theorem 1 we need some lemmas.

LEMMA 1 ([7]). $t^{-1 / 2} \max \left\{\theta_{s} f_{0}:|s| \leq t\right\} \rightarrow 0$ as $t \rightarrow \infty$.

LEMMA 2 ([7]). For any positive constants $c_{1}$,

$$
M_{t+u}-M_{t}=m u(1+o(1))+o(\sqrt{\lambda}), \quad|t| \leq c_{1} \lambda, \quad u \in \mathbf{R},
$$

where $o(1)$ represents a general term that tends to 0 as $\lambda \rightarrow \infty$ uniformly in $(t, u)$ such that $|t| \leq c_{1} \lambda$ and $u \in \mathbf{R}$, for almost all $w ; o(\sqrt{\lambda})$ is a term that can be expressed as $o(1) \sqrt{\lambda}$.

To prove (i) of Theorem 1 it is enough to consider $\int_{0}^{x} \theta_{y} f d y$ by virtue of (0.2). Making habitual use of $t$ to indicate time we write

$$
\frac{1}{\sqrt{\lambda}} \int_{0}^{\lambda t} \theta_{s} f d s=\frac{1}{\gamma \sqrt{\lambda}} \int_{0}^{\lambda t} \theta_{s} f_{0} d w(s)-\frac{1}{\gamma \sqrt{\lambda}}\left(\theta_{\lambda t} f_{0}-f_{0}\right) .
$$

By the ergodicity of $\left\{\theta_{t}\right\}$ and also by (0.4) we see that the quadratic variation of the stochastic integral term in (1.1) tends to $B t / 4$ as $\lambda \rightarrow \infty$, a.s., so the stochastic integral term itself converges in law to $\left\{(B / 4)^{1 / 2} w(t), t \geq 0, P\right\}$ as $\lambda \rightarrow \infty$. The second term of the right hand side of (1.1) is negligible by Lemma 1. Therefore $X_{\lambda}^{+}=\left\{\lambda^{-1 / 2} \int_{0}^{\lambda t} \theta_{s} f d s, t \geq 0\right\}$ converges in law to $\left\{(B / 4)^{1 / 2} w(t), t \geq 0\right\}$, so does $X_{\lambda}^{-}=\left\{\lambda^{-1 / 2} \int_{0}^{-\lambda t} \theta_{s} f d s, t \geq 0\right\}$ because of the reversibility of the diffusion $\theta_{t} f$. Now the assertion (i) of Theorem 1 follows from the fact that $X_{\lambda}^{+}$and $X_{\lambda}^{-}$are asymptotically independent as $\lambda \rightarrow \infty$.

To proceed let $\xi=\lambda m$ and put

$$
\beta_{\lambda}(t)=(B \lambda)^{-1 / 2}\left(M_{\lambda t}-\lambda m t\right), \quad \tilde{\beta}_{\lambda}(t)=\left(m^{-3} B \xi\right)^{-1 / 2}\left(\mu(\xi t)-\xi m^{-1} t\right) .
$$

Then the assertion of (ii) of Theorem 1 follows immediately from the following Lemma.

LEMMA 3. For any $t_{0}>0$ and $\varepsilon>0$

$$
\lim _{\lambda \rightarrow \infty} P\left\{\sup _{|t| \leq t_{0}}\left|\beta_{\lambda}(t)+\tilde{\beta}_{\lambda}(t)\right|>\varepsilon\right\}=0 .
$$


Proof. From the second equality of (1.2) we have $\lambda m t=$ $M\left(\lambda t+\tilde{\beta}_{\lambda}(t) m^{-1} \sqrt{B \lambda}\right)$ and hence

$$
(B \lambda)^{-1 / 2}\left\{M\left(\lambda t+\tilde{\beta}_{\lambda}(t) m^{-1} \sqrt{B \lambda}\right)-M(\lambda t)\right\}=-\beta_{\lambda}(t)
$$

so an application of Lemma 2 yields $\tilde{\beta}_{\lambda}(t)(1+o(1))+o(1)=-\beta_{\lambda}(t)$, where $o(1)$ is a term tending to 0 uniformly on each finite $t$-interval as $\lambda \rightarrow \infty$, a.s. This implies the lemma.

The following observation will lead to another proof of (ii) of Theorem 1. If $v(t)$ denotes the inverse function of $\int_{0}^{x} \theta_{y} f_{0} d y$, then $v(t)=\mu(2 t)$ and the derivative $v^{\prime}(t)$, which equals to $1 / \theta_{v(t)} f_{0}$, is a stationary diffusion process obtained from $\theta_{t} f_{0}$ by changing time and scale.

\section{The proof of Theorem 2}

We give the proof of the part (i). Taking an arbitrary positive sequence $\left\{\lambda_{n}, n=1,2, \ldots\right\}$ tending to $\infty$, we denote by $P^{(n)}$ the probability law of the process $\left\{\lambda_{n}^{-1 / 2}\left(T_{\lambda_{n} x}-\lambda_{n} m x\right), x \geq 0, \mathscr{P}\right\}$. Note that $P^{(n)}$ is a probability measure on the Skorohod space $D=D[0, \infty)$. For the proof of the part (i) it is enough to show that $P^{(n)}$ converges to the probability law of the process $\{\sqrt{C} w(x), x \geq 0, P\}$ as $n \rightarrow \infty$. We first prove that the sequence $\left\{P^{(n)}, n \geq 1\right\}$ is tight. If $Q_{w}^{(n)}$ denotes the probability law of the process $\left\{\lambda_{n}^{-1 / 2}\left(T_{\lambda_{n} x}-M_{\lambda_{n} x}\right)\right.$, $\left.x \geq 0, P_{w}\right\}$, then $Q_{w}^{(n)} \rightarrow Q_{1}\left(P\right.$-a.s.) by Theorem A and hence $Q^{(n)}=\int Q_{w}^{(n)} P(d w)$ also converges to $Q_{1}$ as $n \rightarrow \infty$ where $Q_{1}$ is the probability law (on $D$ ) of the process $\{\sqrt{A} w(x), x \geq 0, P\}$. All the convergence here is to be understood as the convergence of probability measures on $D$. Therefore for any $\varepsilon>0$ there exists a compact set $K_{1} \subset D$ such that $Q^{(n)}\left(K_{1}^{c}\right)<\varepsilon^{2}$ for all $n \geq 1$. We then have $P\left\{L_{n}\right\}<\varepsilon$ where $L_{n}=\left\{w: Q_{w}^{(n)}\left(K_{1}^{c}\right)>\varepsilon\right\}=\left\{w: Q_{w}^{(n)}\left(K_{1}\right) \leq 1-\varepsilon\right\}$. We also introduce, for each fixed $w$, an element $\varphi_{n}(w)$ of $\Omega$ which is defined to be the function $\lambda_{n}^{1 / 2}\left(M_{\lambda_{n} x}-\lambda_{n} m x\right)$ of $x$. Then $P \circ \varphi_{n}^{-1} \rightarrow Q_{2}$ as $n \rightarrow \infty$ by Theorem 1 where $Q_{2}$ is the probability law (on $\Omega$ ) of the process $\{\sqrt{B} w(x)$, $x \geq 0, P\}$. We can thus find a compact set $K_{2} \subset \Omega$ such that $P \circ \varphi_{n}^{-1}\left(K_{2}^{c}\right)<\varepsilon$ for all $n \geq 1$. We now put $K=\left\{w_{1}+w_{2}: w_{1} \in K_{1}, w_{2} \in K_{2}\right\}$. Then $K$ is a compact subset of $D$. Since

$$
\frac{T_{\lambda_{n} x}-\lambda_{n} m x}{\sqrt{\lambda_{n}}}=\frac{T_{\lambda_{n} x}-M_{\lambda_{n} x}}{\sqrt{\lambda_{n}}}+\frac{M_{\lambda_{n} x}-\lambda_{n} m x}{\sqrt{\lambda_{n}}}
$$

we have 


$$
\begin{aligned}
P^{(n)}(K) & =\iint 1_{K}\left(w_{1}+\varphi_{n}(w)\right) Q_{w}^{(n)}\left(d w_{1}\right) P(d w) \\
& \geq \iint 1_{K_{1}}\left(w_{1}\right) 1_{K_{2}}\left(\varphi_{n}(w)\right) Q_{w}^{(n)}\left(d w_{1}\right) P(d w) \\
& =\int_{\varphi_{n}^{-1}\left(K_{2}\right)} Q_{w}^{(n)}\left(K_{1}\right) P(d w) \\
& \geq \int_{\varphi_{n}^{-1}\left(K_{2}\right) \cap L_{n}^{c}}(1-\varepsilon) P(d w) \geq(1-\varepsilon)(1-2 \varepsilon)
\end{aligned}
$$

which proves that $\left\{P^{(n)}, n \geq 1\right\}$ is tight. Therefore, for the proof of Theorem 2 it is enough to show that

$$
\lim _{n \rightarrow \infty} \int f(w) P^{(n)}(d w)=\iint f\left(w_{1}+w_{2}\right) Q_{1}\left(d w_{1}\right) Q_{2}\left(d w_{2}\right)
$$

for any function $f$ of the form

$$
f(w)=\exp \left\{\sqrt{-1} \sum_{j=1}^{k} \alpha_{j} w\left(t_{j}\right)\right\},
$$

where $\alpha_{j} \in \mathbf{R}$ and $t_{j} \geq 0,1 \leq j \leq k$. For such an $f$ the left hand side of $(2.1)$ equals

$$
\begin{aligned}
& \lim _{n \rightarrow \infty} \iint f\left(w_{1}+\varphi_{n}(w)\right) Q_{w}^{(n)}\left(d w_{1}\right) P(d w) \\
& \quad=\lim _{n \rightarrow \infty} \int_{W}\left\{\int_{D} f\left(w_{1}\right) Q_{w}^{(n)}\left(d w_{1}\right)\right\} f\left(\varphi_{n}(w)\right) P(d w) \\
& \quad=\int f\left(w_{1}\right) Q_{1}\left(d w_{1}\right) \int f\left(w_{2}\right) Q_{2}\left(d w_{2}\right)
\end{aligned}
$$

which also equals the right hand side of (2.1). This completes the proof of (i) of Theorem 2.

The part (ii) of Theorem 2 can be proved in a way similar to the above by making use of Theorem $\mathrm{A}$ and Theorem 1.

\section{Supplement to the proof of (i) of Theorem A}

The proof of Theorem A was given in [7]; however, some details in the proof of the part (i) were omitted. It will be worth supplementing them.

The proof of Theorem $\mathrm{A}$ given in [7] proceeds as follows. Let $\tau_{k}=$ 
$T_{k}-T_{k-1}, \tilde{\tau}_{k}=\tau_{k}-E_{w}\left\{\tau_{k}\right\}, k \geq 1$. Then it was proved that, for almost all $w,\left\{\tilde{\tau}_{k}, k \geq 1, \boldsymbol{P}_{w}\right\}$ is a sequence of independent random variables satisfying the Lindeberg condition. Therefore the central limit theorem holds for $T_{n}$ with respect to $P_{w}$, for almost all $w$. Note that $E_{w}\left\{T_{n}\right\}=M_{n}$ and $\operatorname{Var}_{w}\left\{T_{n}\right\} \sim A n$ as $n \rightarrow \infty$ (P-a.s.). Now the rest of the proof, whose detail was omitted in [7], is given as follows.

Let $t_{n k}=\operatorname{Var}_{w}\left\{\tau_{k}\right\} / \operatorname{Var}_{w}\left\{T_{n}\right\}, \zeta_{n k}=(A n)^{-1 / 2}\left\{T_{k}-M_{k}\right\}, 1 \leq k \leq n$, and $t_{n 0}=\zeta_{n 0}=0$. For each fixed $w$ we construct a piece-wise linear function $\xi_{n}(x)$, $0 \leq x \leq 1$, with vertexes $\left(\sum_{j=0}^{k} t_{n j}, \zeta_{n k}\right), 0 \leq k \leq n$. We regard $\left\{\xi_{n}(x)\right.$, $\left.0 \leq x \leq 1, P_{w}\right\}$ as a process with time parameter $x$. Then by Theorem 3.1 of [5], for almost all $w$, the process $\left\{\xi_{n}(x), 0 \leq x \leq 1, P_{w}\right\}$ converges in law to a Brownian motion as $n \rightarrow \infty$. We now modify $\xi_{n}(x)$ slightly, namely, we consider a piece-wise linear function $\eta_{n}(x)$ with vertexes $\left(k / n, \zeta_{n k}\right), 0 \leq k \leq n$. Then $\eta_{n}(x)$ can be represented as $\eta_{n}(x)=\xi_{n}\left(\varphi_{n}(x)\right)$ where $\varphi_{n}(x)$ is the piecewise linear function with vertexes $\left(k / n, \sum_{j=0}^{k} t_{n j}\right), 0 \leq k \leq n$. On the other hand it is easy to see that, for each fixed $x, \varphi_{n} \rightarrow x$ as $n \rightarrow \infty$ for almost all $w$. This combined with the fact that $\varphi$ is increasing implies that $\varphi_{n} \rightarrow x$ uniformly as $n \rightarrow \infty$ (P-a.s.). Therefore

the process $\left\{\eta_{n}(x), 0 \leq x \leq 1, P_{w}\right\}$ converges in law to a Brownian motion as $n \rightarrow \infty$ for almost all $w$.

We finally prove that the process $\left\{(A \lambda)^{-1 / 2}\left(T_{\lambda x}-M_{\lambda x}\right), x \in[0,1], P_{w}\right\}$ converges in law to a Brownian motion as $\lambda \rightarrow \infty$ for almost all $w$; the time interval $[0,1]$ can be replaced by an arbitrary interval $\left[0, t_{0}\right]$ with a minor modification of the proof. Given $x \in(0,1]$ and an integer $n \geq 1$ we take the integer $k$ such that $(k-1) / n<x \leq k / n$. Then $T_{n x}-M_{n x}>T_{k-1}-$ $M_{k}>\sqrt{A n} \eta_{n}(x)-\tau_{k}-m_{k}$ where $m_{k}=M_{k}-M_{k-1}$. Similarly $T_{n x}-M_{n x}<$ $\sqrt{A n} \eta_{n}(x)+\tau_{k}+m_{k}$ and hence

$$
\sqrt{A n} \eta_{n}(x)-\left(\tau_{k}+m_{k}\right)<T_{n x}-M_{n x}<\sqrt{A n} \eta_{n}(x)+\left(\tau_{k}+m_{k}\right) .
$$

This implies that for $x \in[0,1]$

$$
\sqrt{A n} \eta_{n}(x)-\left(\hat{\tau}_{n}+\hat{m}_{n}\right)<T_{n x}-M_{n x}<\sqrt{A n} \eta_{n}(x)+\left(\hat{\tau}_{n}+\hat{m}_{n}\right)
$$

where $\hat{\tau}_{n}=\max \left\{\tau_{k}: 1 \leq k \leq n\right\}$ and $\hat{m}_{n}=\max \left\{m_{k}: 1 \leq k \leq n\right\}$. Next, given $\lambda>0$ let $n=n(\lambda)$ be the integer such that $n-1<\lambda \leq n$. Then $T_{(n-1) x}$ $M_{\lambda x}<T_{\lambda x}-M_{\lambda x} \leq T_{n x}-M_{\lambda x}$, which combined with (3.2) implies 


$$
\begin{aligned}
& \sqrt{A(n-1)} \eta_{n-1}(x)-\left(\hat{\tau}_{n-1}+\hat{m}_{n-1}\right)-\left(M_{\lambda x}-M_{(n-1) x}\right) \\
& \quad<T_{\lambda x}-M_{\lambda x}<\sqrt{A n} \eta_{n}(x)+\left(\hat{\tau}_{n}+\hat{m}_{n}\right)+\left(M_{n x}-M_{\lambda x}\right) .
\end{aligned}
$$

Since $M_{\lambda x}-M_{(n-1) x}$ and $M_{n x}-M_{\lambda x}$ are dominated by $2 \hat{m}_{n}$, we obtain

$$
\begin{gathered}
\left(\frac{n-1}{\lambda}\right)^{1 / 2} \eta_{n-1}(x)-(A \lambda)^{-1 / 2}\left(\hat{\tau}_{n}+3 \hat{m}_{n}\right)<(A \lambda)^{-1 / 2}\left(T_{\lambda x}-M_{\lambda x}\right) \\
<\left(\frac{n}{\lambda}\right)^{1 / 2} \eta_{n}(x)+(A \lambda)^{-1 / 2}\left(\hat{\tau}_{n}+3 \hat{m}_{n}\right) .
\end{gathered}
$$

On the other hand we can prove that for almost all $w$

$$
\begin{gathered}
P_{w}\left\{\lim _{n \rightarrow \infty} \hat{\tau}_{n} / \sqrt{n}=0\right\}=1, \\
\lim _{n \rightarrow \infty} \hat{m}_{n} / \sqrt{n}=0 .
\end{gathered}
$$

In fact, it is easy to see that $\left\{\tau_{k}, k \geq 1, \mathscr{P}\right\}$ is stationary and ergodic. Since $\tau_{k}^{2}$ is integrable we have $n^{-1} \sum_{1}^{n} \tau_{k}^{2} \rightarrow$ const. as $n \rightarrow \infty\left(\mathscr{P}_{\text {-a.s. }) \text { and hence }}\right.$

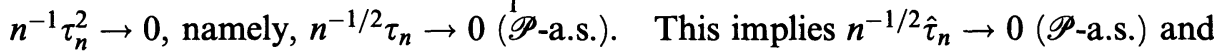
hence (3.4). (3.5) can be proved in a similar manner. By virtue of (3.1), (3.4) and (3.5) the processes of the leftmost and rightmost hands of (3.3) converge in law to a Brownian motion as $\lambda \rightarrow \infty$. Therefore (3.3) implies the assertion for $(A \lambda)^{-1 / 2}\left(T_{\lambda x}-M_{\lambda x}\right)$ that we wanted to prove.

\section{References}

[1] K. Itô and H. P. McKean, Diffusion Processes and Their Sample Paths, Springer-Verlag, New York, 1965.

[2] K. Kawazu and H. Tanaka, A diffusion process in a Brownian environment with drift, J. Math. Soc. Japan 49 (1997), 189-211.

[3] H. Kesten, M. V. Kozlov and F. Spitzer, A limit law for random walk in a random environment, Composito Math. 30 (1975), 145-168.

[4] Yu. V. Prohorov, Convergence of random processes and limit theorems in probability theory, Theor. Probab. Appl. 1 (1956), 157-214.

[5] F. Solomon, Random walks in a random environment, Ann. Probab. 3 (1975), 1131.

[6] H. Tanaka, Diffusion processes in random environments, Proc. International Congress of Mathematicians, Zürich, 1994, 1047-1054, Birkhäuser Verlag, Basel, 1995.

[7] H. Tanaka, Environment-wise central limit theorem for a diffusion in a Brownian environment with large drift, Itô's Stochastic Calculus and Probability Theory, ed. by N. Ikeda et al., pp. 373-384, Springer, 1996. 
[8] H. Tanaka, Limit theorems for a Brownian motion with drift in a white noise environment, Chaos, Solitons \& Fractals, 8 (1997), 1807-1816.

\author{
K. Kawazu \\ Department of Mathematics \\ Faculty of Education \\ Yamaguchi University \\ Yosida, Yamaguchi 753-8513 \\ Japan \\ H. Tanaka \\ Department of Mathematics \\ Faculty of Science and \\ Technology \\ Keio University \\ Yokohama 223-0061 \\ Japan
}


